\title{
Antiviral polymers based on N-halamine polyurea
}

Noam Y. Steinman ${ }^{1}$, Tan Hu ${ }^{2,4}$, Aviv Dombrovsky ${ }^{3}$, Meital Reches ${ }^{2}$ and Abraham J.

$$
\operatorname{Domb}^{1 *}
$$

1 The Alex Grass center for Drug Design and Synthesis and Center for Cannabis

Research and the Institute of Drug Research, School of Pharmacy-Faculty of Medicine, The Hebrew University of Jerusalem, Jerusalem, 91120, Israel

2 Institute of Chemistry and The Center for Nanoscience and Nanotechnology, The Hebrew University of Jerusalem, Edmond J. Safra Campus, Givat Ram, Jerusalem 9190401, Israel

3 Department of Plant Pathology and Weed Research, Institute of Plant Protection, Volcani Center, ARO, Israel

4 College of Food Science and Technology, Huazhong Agricultural University, Wuhan, Hubei 430070, PR China; Key Laboratory of Environment Correlative Dietology, Huazhong Agricultural University, Ministry of Education, Wuhan, Hubei 430070, PR China 
Supporting Information

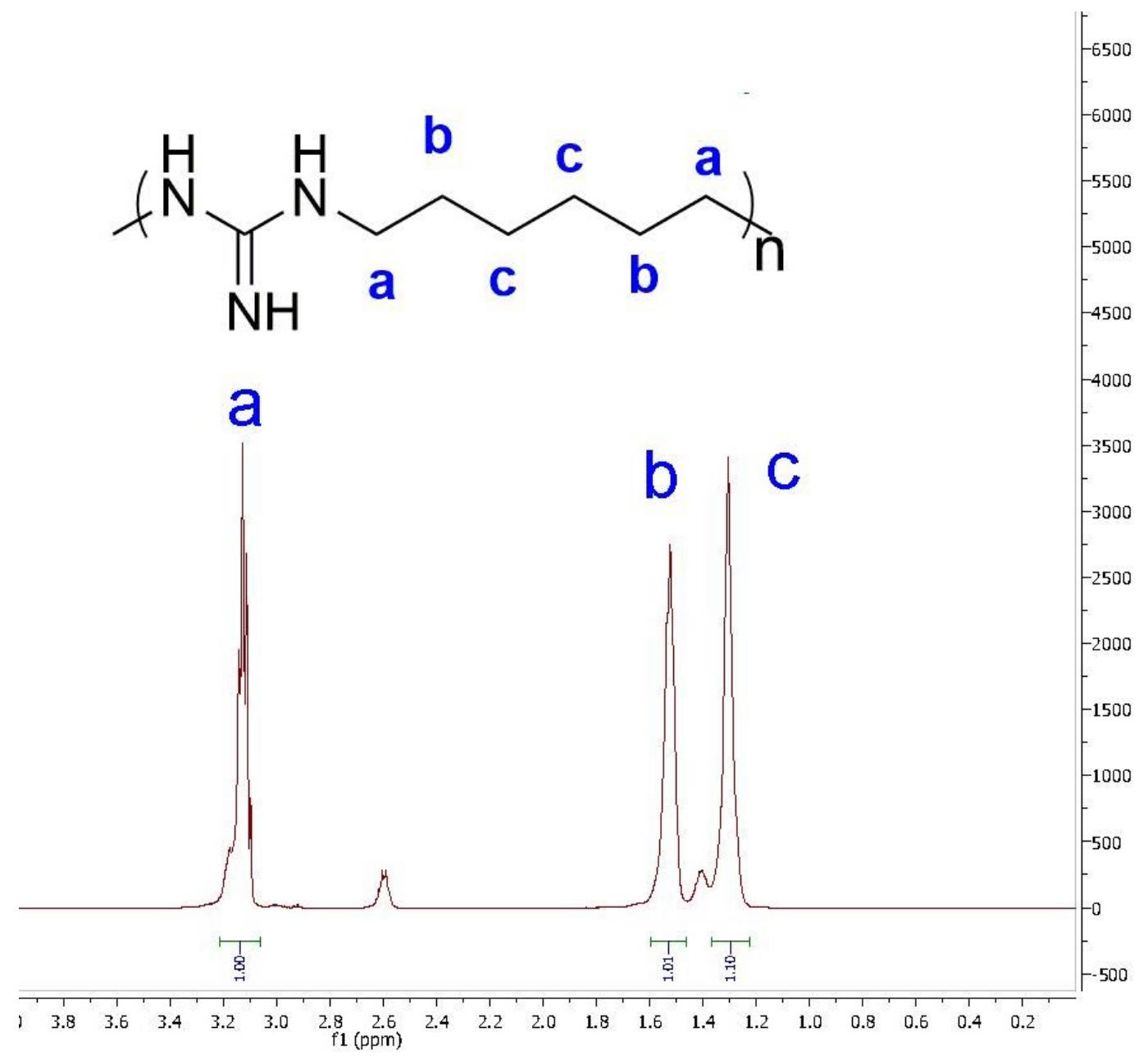

Figure S1. ${ }^{1} \mathrm{H}$ NMR spectrum of PHMG with peak assignments. 


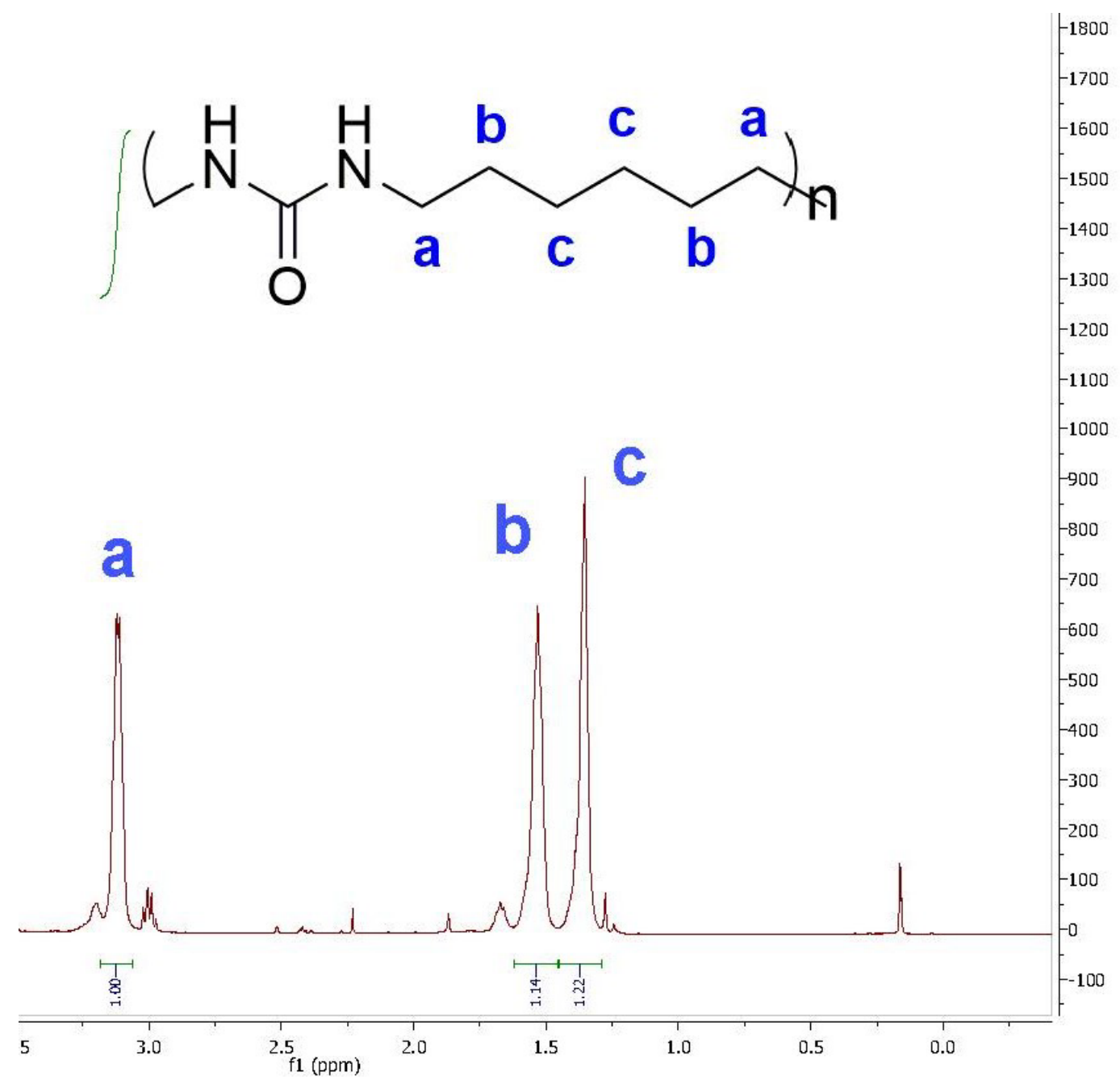

Figure S2. ${ }^{1} \mathrm{H}$ NMR spectrum of PHMU with peak assignments. 


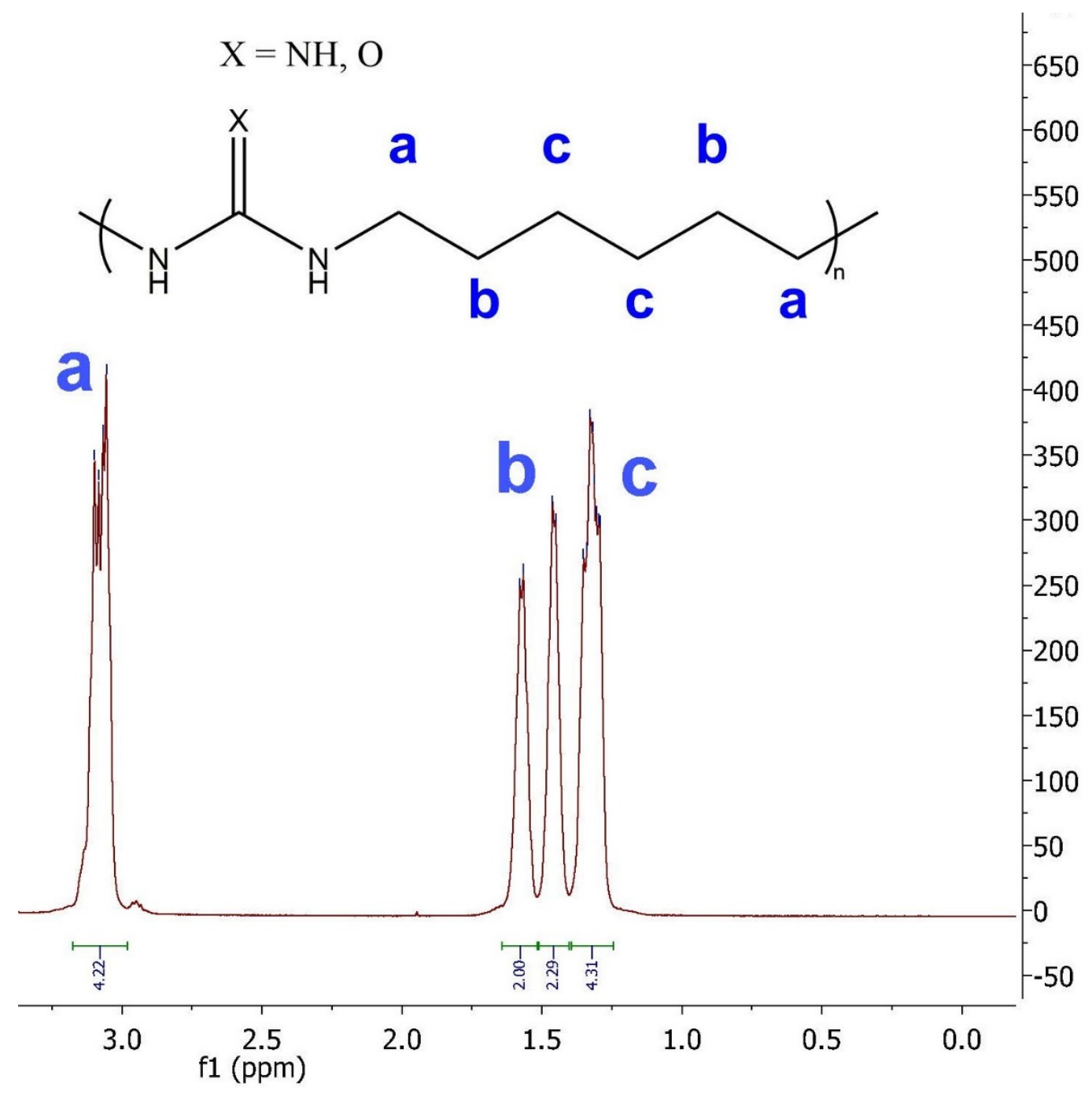

Figure S3. ${ }^{1} \mathrm{H}$ NMR spectrum of PHMGU with peak assignments. 


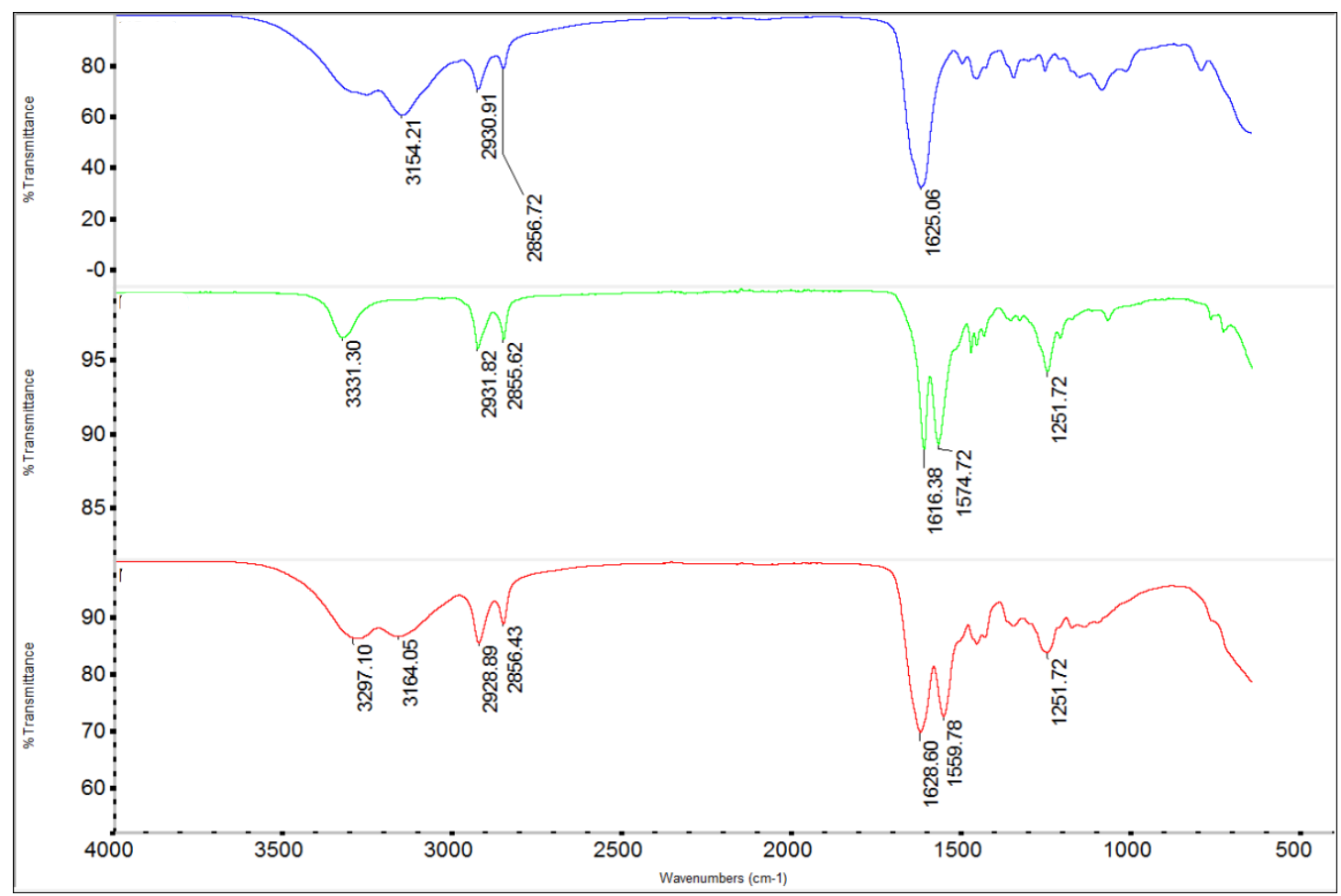

Figure S4. IR spectrum of PHMG (top), PHMU (middle) and PHMGU (bottom). 


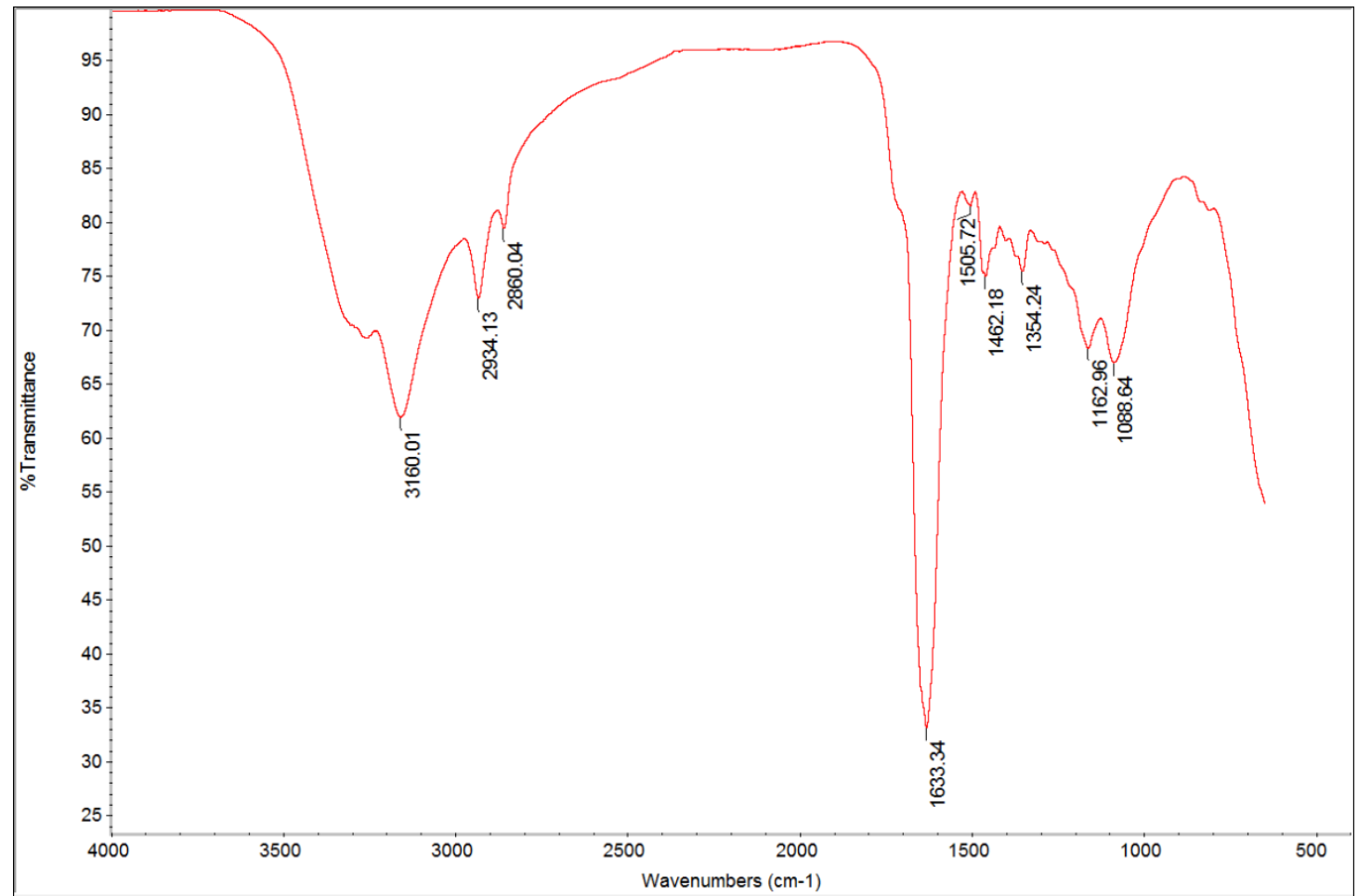

Figure S5. IR spectrum of chlorinated PHMG.

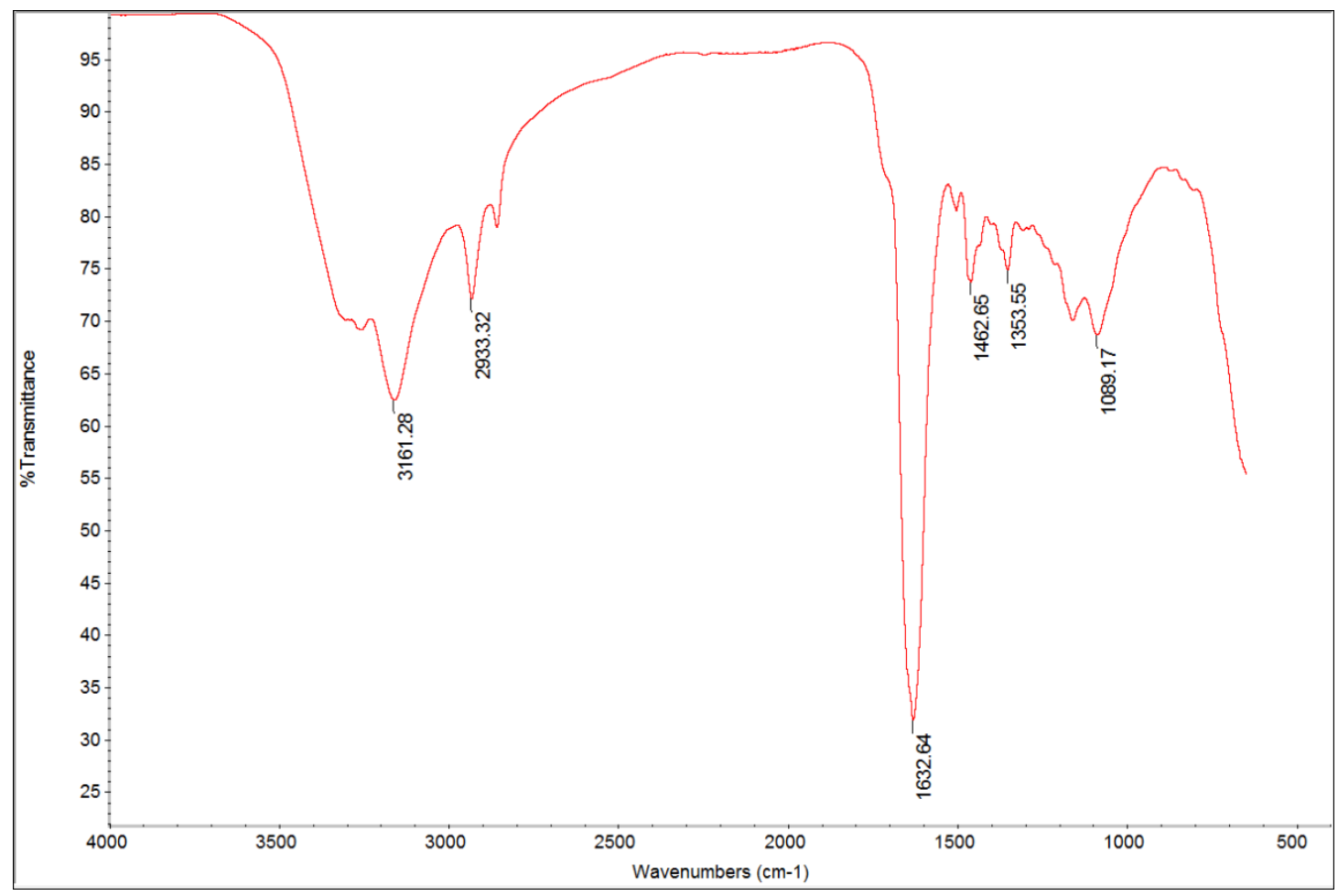

Figure S6. IR spectrum of brominated PHMG. 


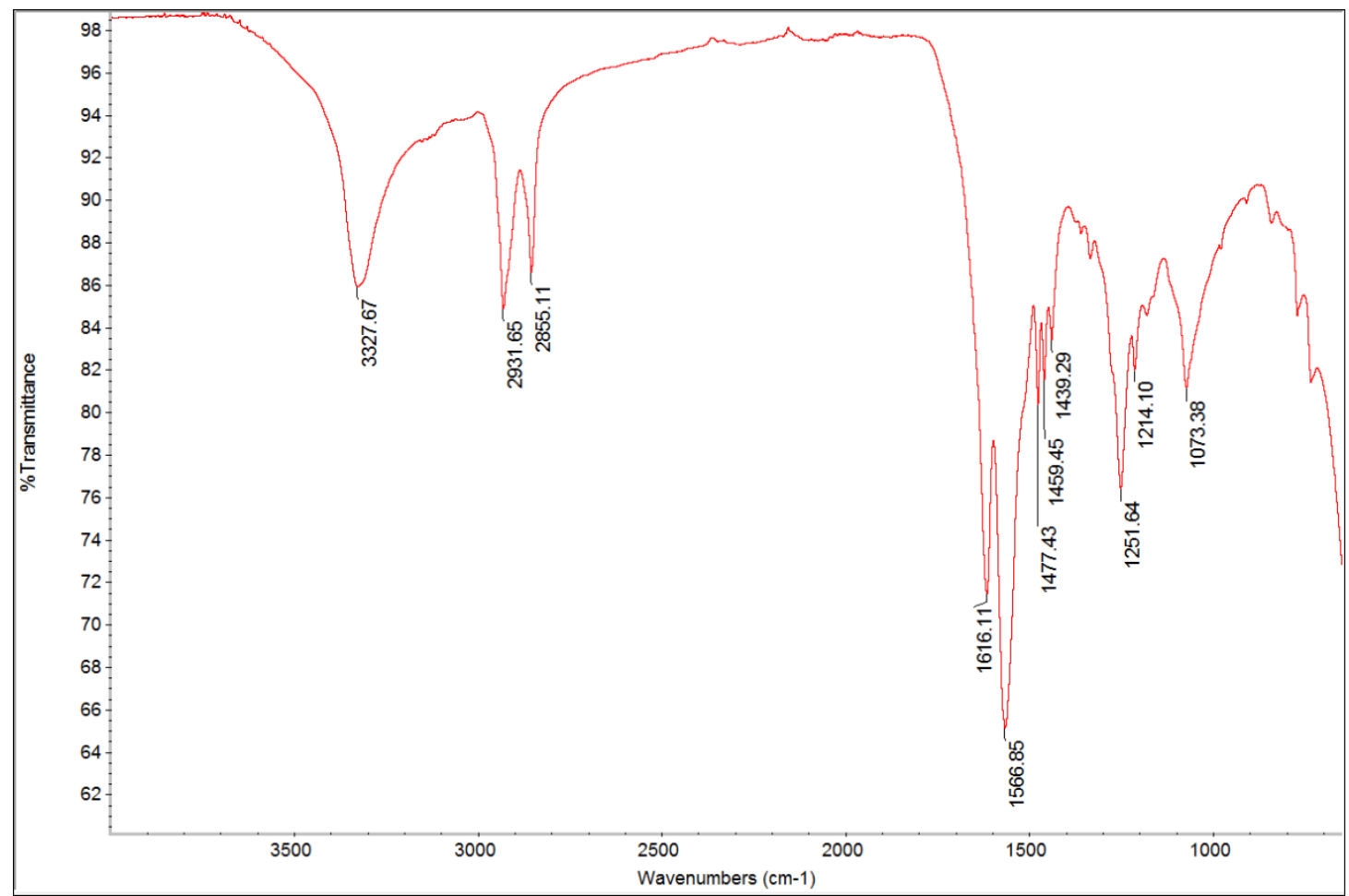

Figure S7. IR spectrum of chlorinated PHMU.

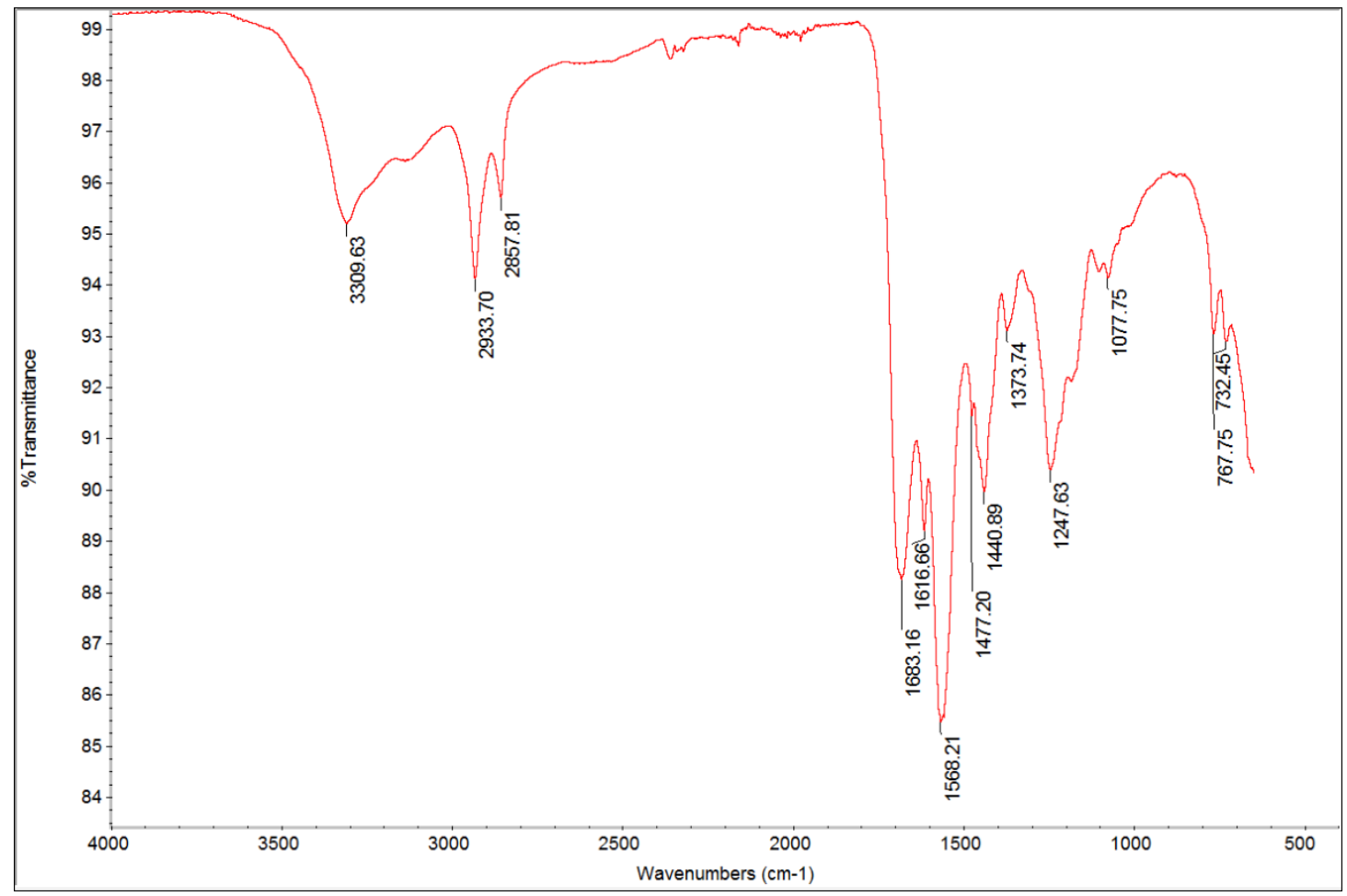

Figure S8. IR spectrum of brominated PHMU. 


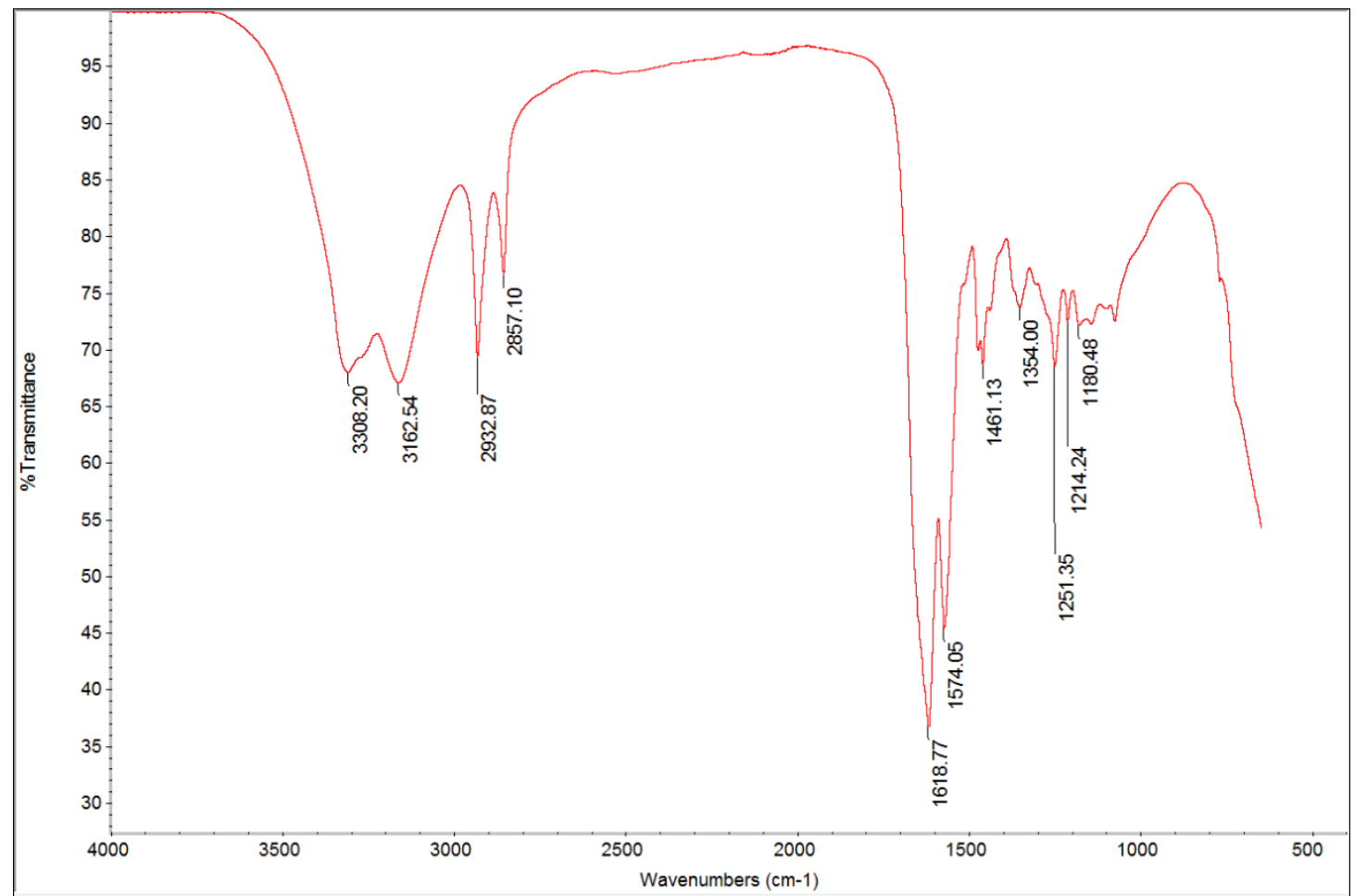

Figure S9. IR spectrum of chlorinated PHMGU.

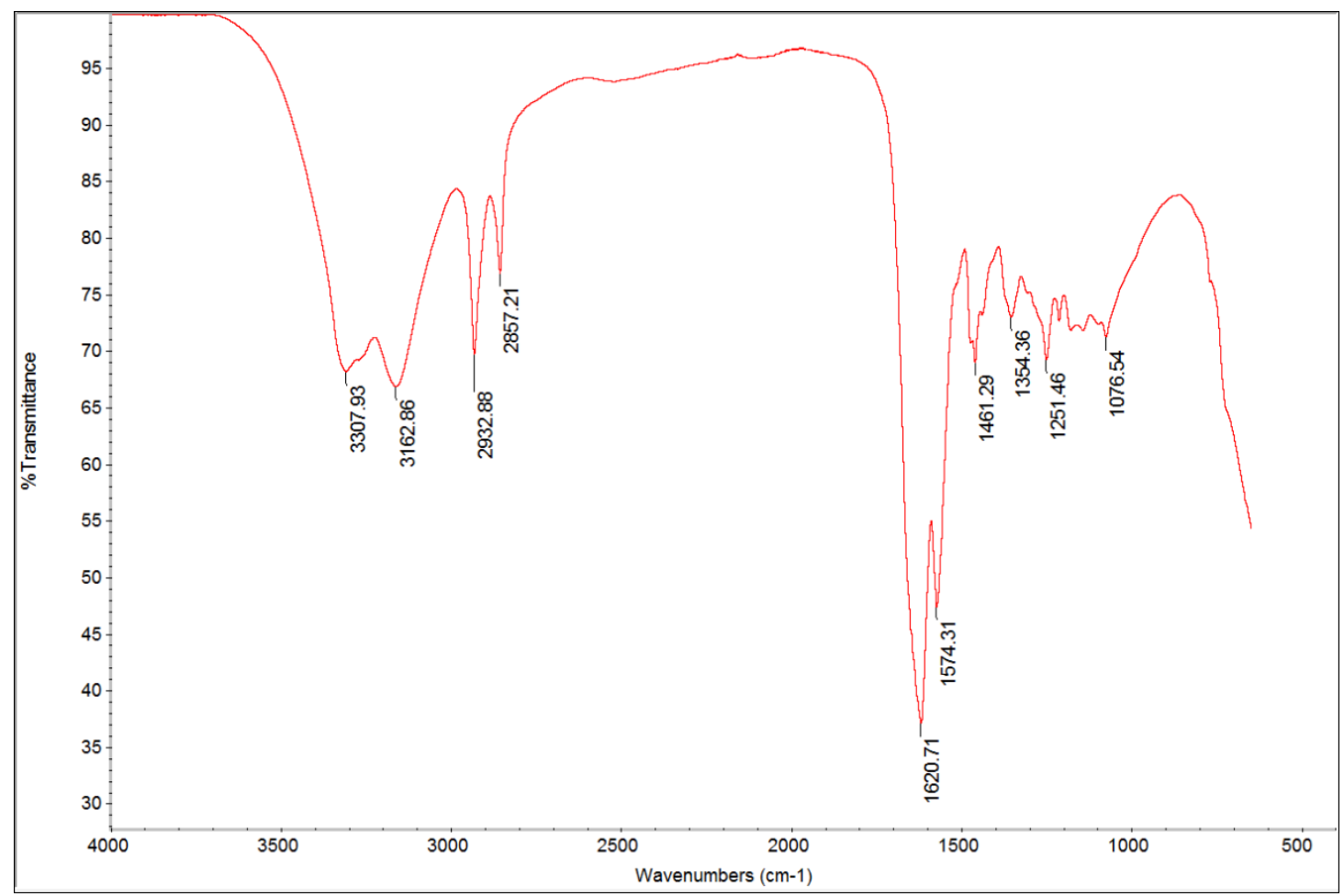

Figure S10. IR spectrum of brominated PHMGU. 